\title{
Editorial: Applications of Genome Sequences for Discovering Characteristics that Are Unique to Different Groups of Organisms and Provide Insights into Evolutionary Relationships
}

\author{
Radhey S. Gupta * \\ Department of Biochemistry and Biomedical Sciences, McMaster University, Hamilton, ON, Canada
}

Keywords: comparative genomics, microbial classification, molecular signatures, genetic and biochemical characteristics, conserved signature indels

\section{The Editorial on the Research Topic}

Applications of Genome Sequences for Discovering Characteristics that are Unique to Different Groups of Organisms and Provide Insights into Evolutionary Relationships

\section{OPEN ACCESS}

Edited and reviewed by: John R. Battista,

Louisiana State University, USA

*Correspondence:

Radhey S. Gupta

gupta@mcmaster.ca

Specialty section:

This article was submitted to

Evolutionary and Genomic

Microbiology,

a section of the journal

Frontiers in Genetics

Received: 03 February 2016 Accepted: 08 February 2016 Published: 19 February 2016

Citation:

Gupta RS (2016) Editorial: Applications of Genome Sequences for Discovering Characteristics that

Are Unique to Different Groups of Organisms and Provide Insights into

Evolutionary Relationships.

Front. Genet. 7:27.

doi: 10.3389/fgene.2016.00027
Genome sequences are making available an unprecedented amount of genetic information that has the potential to reliably elucidate many aspects of physiology, biochemistry, and evolutionary relationships of different organisms. For efficient analyses of the vast amount of genomic sequence data, new reductive approaches are needed which can identify reliable genetic characteristics that are specific for either particular species or related groups of organisms. These characteristics provide novel means for distinguishing different organisms and for understanding their evolutionary history as well as novel tools for phenotypic, behavioral, and biochemical studies. The papers collected in this research topic discuss the application of comparative genomic techniques in a wide range of modalities covering the evolution, classification, identification, and characterization of bacteria and viruses.

The evolutionary relationships of bacterial organisms form the underlying basis of their classification. Koton et al. utilize genomic sequence data to elucidate the evolutionary interrelationships of Vibrio vulnificus strains and to both trace the origin and identify unique genomic characteristics of $V$. vulnificus biotype 3 . The comparative genomic analyses completed by Koton et al. are a model of how to utilize the comparative analysis of core, accessory, and unique genomic elements in order to understand the interrelationships of various strains of a single bacterial species. Bacterial classification at the genus and species level, however, is highly structured and often based on the characterization of a limited number of strains (often a single isolate) focussed on the 16S rRNA gene and a number of phenotypic and chemotaxonomic properties (Tindall et al., 2010). Sutcliffe argues that the current standards for classification have limited utility, are time consuming to perform, and are inadequate to face the exponentially growing number bacterial isolates being discovered. He suggests that future classification should focus on utilizing genome sequence data and genome derived characteristics in order to define and delineate bacterial groups. Sawana et al. provide a practical example of this type of genome-centric classification. In their study they utilize genome sequence data to identify multiple highly specific molecular signatures or characteristics which differentiate two main groups within the large and diverse genus 
Burkholderia. They then divide the genus Burkholderia into two genera based on these genome-derived characteristics (viz. Burkholderia and Paraburkholderia). This group of researchers has previously applied similar techniques to divide the genus Borrelia into two genera, Borrelia and Borreliella (Adeolu and Gupta, 2014), and the genus Thermotoga into two genera, Thermotoga and Pseudothermotoga (Bhandari and Gupta, 2014). The benefits of basing classification on genome sequence analysis are numerous. Firstly, genome sequence data is more easily and consistently comparable between different organisms than phenotypic or chemotaxonomic properties which allows for more reliable and consistent delineation between organisms. Secondly, genetic and molecular characteristics universally shared by a defined group of organisms are more stable over time than chemotaxonomic characteristics and are unique to the group for which they were identified facilitating their use in the identification and classification of novel members of the group. Lastly, the identified genomic and molecular differences form the basis of any recognized or unrecognized phenotypic or biochemical differences between the different groups of organisms, thus, they are important novel targets of study and should lead to a greater focus on understanding of the underlying biological differences between the indicated groups. The utility of comparative genomic analysis in studying the underlying biological differences between groups of organisms is exemplified by Yang et al. who utilize the comparative analysis of Microcystis aeruginosa genomes to identify the CRISPR-Cas systems of different Microcystis aeruginosa subgroups, giving novel insights into their resistance to bacteriophages and their ability to modulate genomic stability and plasticity. Liu et al. utilize a number of biochemical assays to characterize the function of a laterally transferred gene cluster containing the tet $R$ gene, identified via comparative genomic techniques. They show that the tet $R$ gene and TetR protein contribute to cell survival under oxidative stress and are able to identify a number of regulatory roles played by the TetR protein. Taken together, these two studies exemplify the utility of studying and characterizing the genome derived characteristics that are beginning to be used to classify bacterial groups.

Viruses and virus-like particles represent the most abundant sources of DNA in the environment (Weinbauer, 2004). Due to the size, plasticity, and unique composition of viral

\section{REFERENCES}

Adeolu, M., and Gupta, R. S. (2014). A phylogenomic and molecular marker based proposal for the division of the genus Borrelia into two genera: the emended genus Borrelia containing only the members of the relapsing fever Borrelia, and the genus Borreliella gen. nov. containing the members of the Lyme disease Borrelia (Borrelia burgdorferi sensu lato complex). Anton. Leeuw. Int. J. G. 105, 1049-1072. doi: 10.1007/s10482-0140164-x

Bhandari, V., and Gupta, R. S. (2014). Molecular signatures for the phylum (class) Thermotogae and a proposal for its division into three orders (Thermotogales, Kosmotogales ord. nov. and Petrotogales ord. nov.) containing four families (Thermotogaceae, Fervidobacteriaceae fam. nov., Kosmotogaceae fam. nov. and Petrotogaceae fam. nov.) and a new genus Pseudothermotoga gen. nov. with five new combinations. Anton. Leeuw. Int. J. G. 105, 143-168. doi: 10.1007/s10482013-0062-7 genomes, they represent particularly interesting candidates for comparative analysis. Despite this, viral genome are relatively unexplored in comparison to both eukaryotic and prokaryotic genomes. Chan et al. use genome sequence data to reveal the evolutionary relationships between the members of N4-like phage genus and to define the core genome of all members of the N4-like phage genus and the core genome of the N4-like Roseobacter phages, providing novel insight into the traits which characterize the genus and their evolutionary relationships. Mohiuddin and Schellhorn utilize environmental sampling and comparative genomic analyses of environmental DNA and DNA extracted from virus-like particles to examine the abundance and distribution of different classes of viruses in freshwater environments. These studies both represent useful applications of genomic sequence data and genome derived characteristics to identify and characterize viral groups and populations that are currently underexplored.

The papers presented in this research topic exemplify the broad range of applications of comparative genomic analysis and genome derived characteristics in biology and related sciences. The use of genome sequence data to answer novel questions or challenge previously held assumptions is revolutionizing all fields of biology and novel methods and applications of comparative genomics are becoming an indispensable part of modern biological research.

\section{AUTHOR CONTRIBUTIONS}

RG was the Editor for this Research Topic. I have critically read all of the papers submitted under this research topic and have written/finalized the submitted Editorial.

\section{ACKNOWLEDGMENTS}

The expert assistance provided by my co-worker Mobolaji Adeolu in writing a draft of this commentary is thankfully acknowledged. Mobolaji could not be included as a co-author in this work as the authorships in the Editorial are limited to the Topic(s) Editor. RG's work in this area was supported by the Natural Sciences and Engineering Research Council of Canada and MRI-ORF Water Round research grants.

Tindall, B. J., Rosselló-Móra, R., Busse, H. J., Ludwig, W., and Kämpfer, P. (2010). Notes on the characterization of prokaryote strains for taxonomic purposes. Int. J. Syst. Evol. Microbiol. 60(Pt 1), 249-266. doi: 10.1099/ijs.0.016949-0

Weinbauer, M. G. (2004). Ecology of prokaryotic viruses. FEMS Microbiol. Rev. 28, 127-181. doi: 10.1016/j.femsre.2003.08.001

Conflict of Interest Statement: The author declares that the research was conducted in the absence of any commercial or financial relationships that could be construed as a potential conflict of interest.

Copyright (c) 2016 Gupta. This is an open-access article distributed under the terms of the Creative Commons Attribution License (CC BY). The use, distribution or reproduction in other forums is permitted, provided the original author(s) or licensor are credited and that the original publication in this journal is cited, in accordance with accepted academic practice. No use, distribution or reproduction is permitted which does not comply with these terms. 